\title{
$26^{\text {th }}$ International Conference \\ ENGINEERING MECHANICS 2020
}

Brno, Czech Republic, November 24 - 25, 2020

\section{NEW METHOD FOR ON-BOARD CAR SUSPENSION TESTING}

\author{
Jeniš F.*, Strecker Z., Mazůrek I.
}

\begin{abstract}
This paper deals with a new automotive suspension testing methodology. The methodology is based on monitoring of the car body movement by inertial measuring unit after crossing a defined obstacle. The attenuation of all suspensions, which serves as information on the shock absorbers condition, is analyzed from the recorded movement. The relative attenuation is evaluated using standardized differentiation of measured quantities and their exponential envelope. The methodology is based on the TriStar tester. The difference of TriStar is especially a manual excitation and measurement of car body movement for each wheel individually. The proposed methodology is simple and time-saving. Experimental verification on a larger sample of vehicles showed that the methodology can detect a vehicle with a damaged damping system. However, it is not sufficiently exact to accurately determine the condition of individual shock absorber, but it is an affordable price for its cost and simplicity. The methodology will find application wherever it is necessary to find and exclude vehicles with critically low damping from traffic.
\end{abstract}

Keywords: Car, Suspension, Tester, On board, Over-cross, Inercial measurement unit.

\section{Introduction}

Recently, more and more emphasis is placed on the safety of automobile traffic. One of the important parts of the vehicle affecting its safety is the shock absorber. The shock absorbers are subject to wear over time, which negatively affects the behavior of the car. Defective shock absorbers cause a longer braking distance, reduce ABS function, reduce vehicle maneuverability and increase wear on suspension components, etc. The shock absorbers wear gradually, so the driver does not register vehicle behavior changes and may be surprised when crisis situation occurs. For this reason, it is necessary to check the function of the shock absorbers at certain intervals. Either after some time or after driving a given number of kilometers.

The most reliable method is to test the removed shock absorber on a special stand, which is, however, very time-consuming and slightly technically demanding. In practice, therefore, mainly non-removing methods are used. These are active resonance testers or passive impulse-attenuation testers. The most widespread is the EUSAMA resonance method, which was introduced in 1976 with the aim of unifying the testing procedure. However, it currently has a problem. It is starting to fail due to the use of low profile tire types and modern chassis adjustment (Klapka, 2016). Impulse-attenuation testers such as TriStar or M-Tronic (Malmedahl, 2005) have the problem of exciting vehicles with recently used rigid suspension.

Due to the planned including of diagnostics of shock absorbers on Periodical technical inspections, it is necessary to solve this situation. The solution may be an improvement of some existing method or a development of new ones. Klapka (2017) proposed extending the EUSAMA methodology to include phase shift evaluation. Peterka (2007) dealt with evaluating a shock absorber by transfer rate between unsprung and sprung mass when crossing an obstacle. This paper deals with the development and validation of the methodology based on the obstacle crossing and the analysis of the suspension relative attenuation.

\section{Materials and Methods}

In this method, the vehicle 5 times crosses the obstacle with a height of $0.08 \mathrm{~m}$ and a length of $0.43 \mathrm{~m}$ with the left and then the right wheels (Fig. 1 left). To ensure repeatability, it is essential that the vehicle maintains the same speed during all test drives. Therefore test drive speed corresponds to idle with the first gear engaged. During the crossing, an inertial measuring unit (IMU) is located behind the driver's seat

All authors: Institute of Machine and Industrial Design, Brno University of Technology, Technická 2898/2, 61669 Brno; CZ, Filip.Jenis@vutbr.cz 
as close to the vehicle gravity center as possible (Fig. 1 right). IMU senses the vertical acceleration of the car body and its angular velocity around the longitudinal and transverse axes (Fig. 2 left). With the known wheelbase, gauge and position of the IMU, the vertical acceleration of the car body at the suspension points is then calculated according to the equation:

$$
a_{i}=a_{z}+(-1)^{i} y_{i} \alpha_{x}+(-1)^{i} x_{i} \alpha_{y}
$$

where: $a_{i}$ - vertical car body acceleration at the point of the $\mathrm{i}^{\text {th }}$ suspension, $a_{z}$ - vertical car body acceleration at the IMU point, $y_{i}$-transverse distance of the $\mathrm{i}^{\text {th }}$ suspension and IMU, $x_{i}$ - longitudinal distance of the $\mathrm{i}^{\text {th }}$ suspension and IMU, $\alpha_{x}$ - angular acceleration of the car body around the longitudinal axis, $\alpha_{y}$ - angular acceleration of the body around the transverse axis (Fig. 2 left).
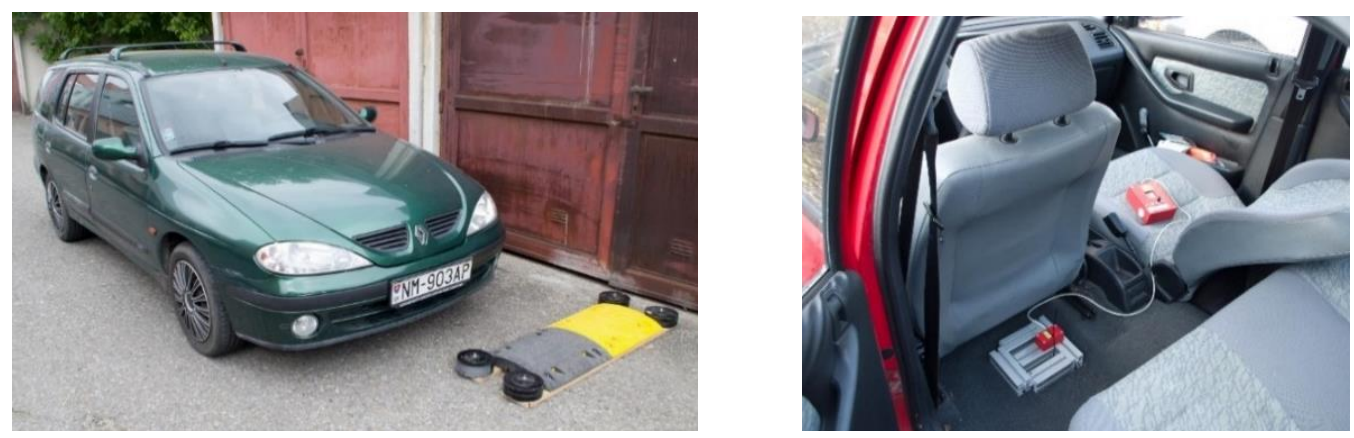

Fig. 1: Car and obstacle during the on board test (left) location of the IMU in the vehicle (right).

From the resulting accelerations, the relative attenuation for each suspension is evaluated. The method developed for this is called the standardized differentiation method. The acceleration is integrated to achieve: displacement and velocity and also derived to obtain jerk. These quantities are phase shifted, therefore in the evaluated part of signal we obtain further amplitudes for analysis (Fig. 2 right). The envelope is then formed from the quantities courses, and its equation exponent represents the system attenuation coefficient:

$$
y=A_{0} e^{-b t}
$$

where: $A_{0}$ - initial displacement, $b$-attenuation coefficient, $t$ - time.
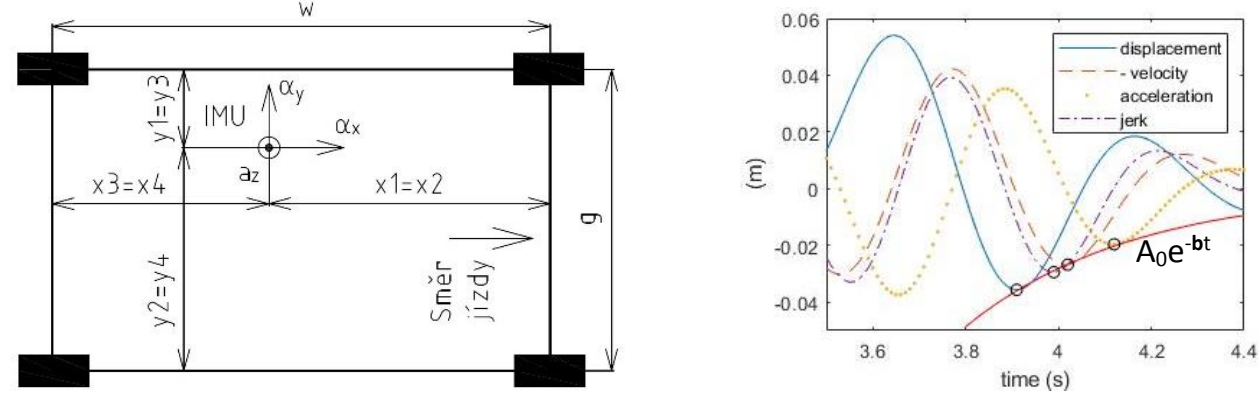

Fig. 2: IMU location schema (left) standardized derivation schema (right).

To create an envelope, the quantities must have the same physical dimension. Therefore, it is necessary to divide/multiply them by the nth power of the natural angular velocity $\omega$, where $\mathbf{n}$ is the number of differentiation/integration, because:

$$
y=A \cdot \sin (\omega t+\varphi), \dot{y}=\omega \cdot y
$$

where: $y$-displacement, $\dot{y}$ - velocity, $A$ - amplitude, $\omega$ - angular velocity, $\varphi$ - phase shift.

The system relative attenuation is the ratio of the attenuation coefficient to the natural angular velocity:

$$
b_{r}=\frac{b}{\omega}
$$

where: $b_{r}$ - relative attenuation, $b$ - attenuation coefficient, $\omega$ - angular velocity.

The resulting value is then the average of 5 test drives. Thanks to this method, attenuation can be evaluated from only one damper extension, which is crucial because the car body oscillation stabilizes very quickly and therefore, due to lack of amplitudes, attenuation cannot be evaluated by logarithmic decrement. Experimental verification of the new methodology was carried out on a set of 65 vehicles. The vehicles 
were tested by the proposed and reference methodology. It was evaluated whether the reference methodology would evaluate the same differences between the shock absorbers as the on board test and whether the vehicles with good or bad damping would be evaluated identically by both methods. Absolute attenuation values were not compared. A commercial TriStar Shock Tester similar to M-Tronic (Malmedahl, 2005) was chosen as the reference methodology. The tester measures the displacement of the car body by the resistance angle sensor. The sensor arm was inserted under the wheel arch and the vehicle was manually excited at the point of the suspension to be analyzed (see Fig. 3). Thus, the car body vertical displacement course at the desired point was recorded. The device measures the maximum velocity of the shock absorber expansion to ensure the valid test conditions. The damper velocity must be within the range of 0.2 to $0.3 \mathrm{~ms}^{-1}$. The standardized differentiation method was also used for attenuation analysis.

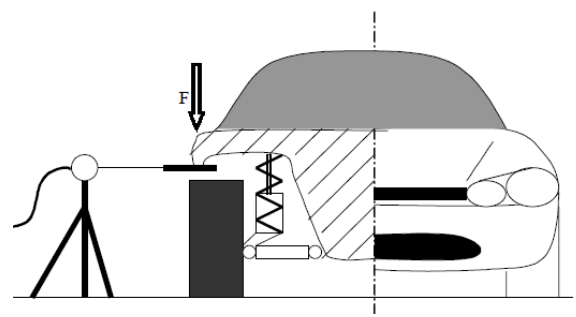

Fig. 3: Principle of TriStar shock tester measurement.

\section{Results}

In Fig. 4, we can see that for some vehicles the methods give satisfactory results and for some vehicles they do not. In the left graph, the variations between the wheels are almost the same for both methods. In the right graph, the difference between the front wheel attenuation deviations is $23 \%$, the rear wheels $11 \%$, and for axles as a whole are $47 \%$. The average deviation difference is $13 \%$ for the front wheels, $8 \%$ for the rear and $18 \%$ for the axles.
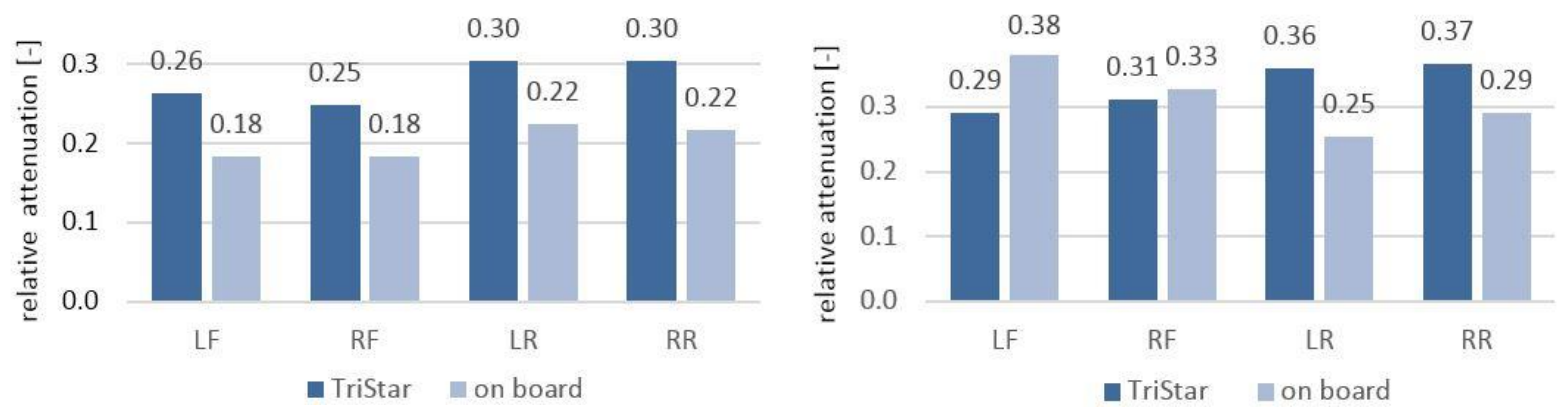

Fig. 4: Suspensions relative attenuation comparison for TriStar and on board tester.

Furthermore, vehicles were compared as a whole. The relative attenuation of each suspension was averaged in a single number for each vehicle for both the TriStar and the on board tester. It can be seen in Fig. 5 that both methods follow the same trend, but the variance of the on board test results is relatively large. It can be expressed as a confidence value $\mathrm{R}^{2}=0.55$. For illustration, an ideal line $y=x$ is plotted (- - ).

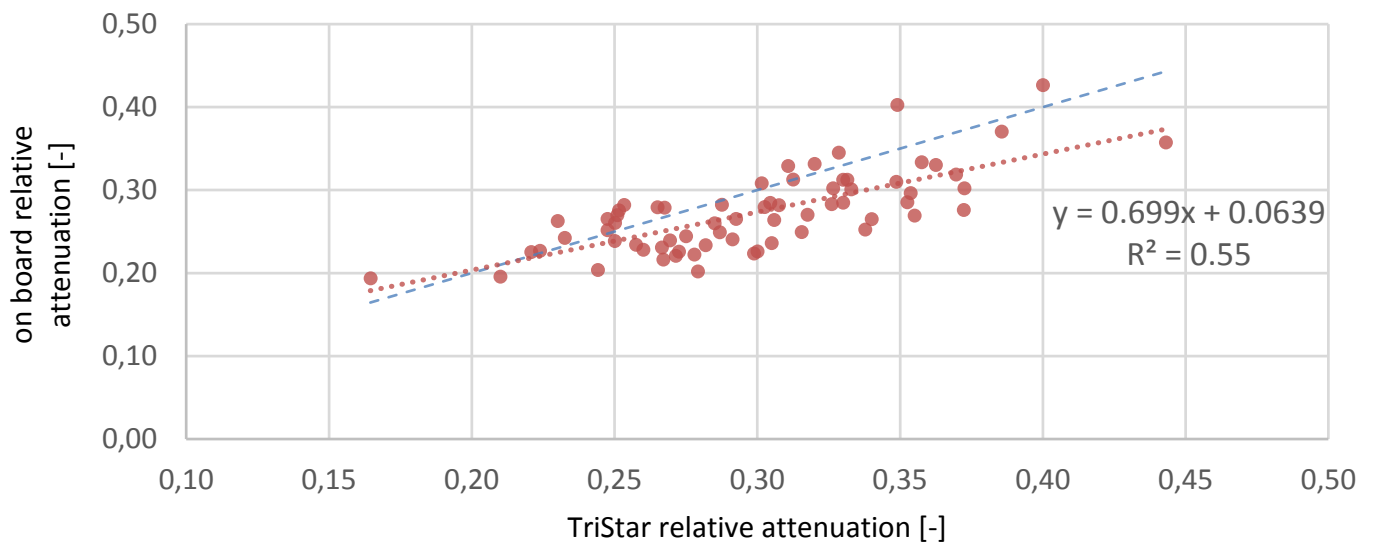

Fig. 5: Results comparison for TriStar and on board tester. 
The method is, however, reliable when a shock absorber is completely out of service. The following graph shows a comparison of default state and state with the RR shock absorber disconnected of a randomly selected Škoda Roomster. Obviously, the method has easily identified the defective shock absorber. It may be noted that along with the decrease in RR suspension attenuation, the attenuations of other suspensions have also decreased. This is logical because the car is a complex system and the movement of its body is dampened by all the shock absorbers at the same time.

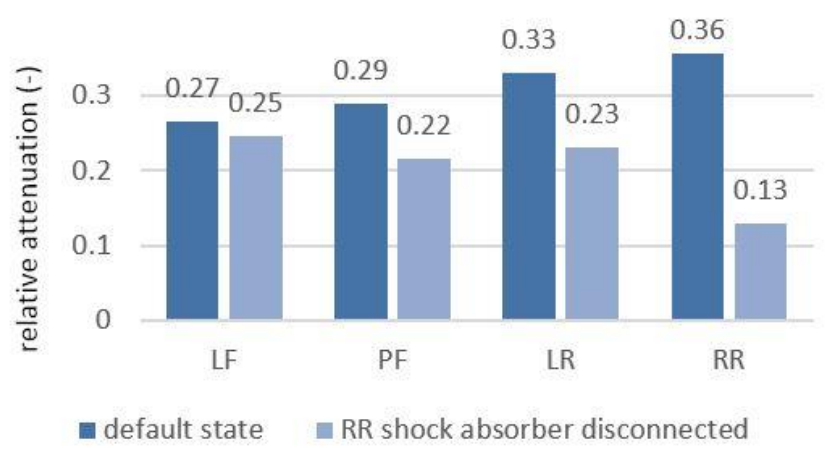

Fig. 6: Comparison for default state and RR shock absorber disconnected.

\section{Conclusions}

The inconvenience of the on-board test is the difficulty in controlling the expansion velocity of the shock absorber during the test. Therefore, each damper is measured under different conditions, which causes a higher dispersion of the results. For the repeatability of the test, it is necessary to maintain the same vehicle speed for all test drives, which is practically not possible other than driving at idle in first gear. Regardless, the vehicle speed varies between 7 to $11 \mathrm{~km} / \mathrm{h}$ for various vehicles. The speed difference in combination with the various suspension parameters (weight, stiffness) causes different shock absorber expansion velocity. The tested system is not linear, so the difference in this velocity causes different attenuation results. TriStar tester monitors the shock absorber expansion velocity and it can be easily corrected with either a higher or lower excitation force.

The on board test was designed as an inexpensive and less accurate alternative to the EUSAMA methodology used today. Its advantages are simplicity, small space and low price. The results show that for some vehicles it is possible to achieve a very good conformity between the TriStar and on board tester. Some of vehicles show a relatively large dispersion. Therefore, the evaluation of relative attenuation may not be sufficiently reliable, but the binary evaluation that is most accessible to service technicians is accurate sufficiently.

\section{Acknowledgement}

We highly appreciate the kind sponsorship of the Faculty of Mechanical Engineering, BUT, which granted as much as they could. The research leading to these results has received funding from FSI-S-20-6247.

\section{References}

Klapka, M., Mazůrek, I. et al. (2016) Twilight of the EUSAMA diagnostic methodology. Meccanica, 52, 9, pp. 2023-2034.

Klapka, M., Mazůrek, I. et al. (2017) Reinvention of the EUSAMA diagnostic methodology. International Journal of Vehicle Design, 74, 4, pp. 304-318.

Malmedahl, G. et al. (2005). Analysis of automotive damper data and design of a portable measurement system. SAE Technical Papers.

Peterka, B. (2007) Diagnostics of shock absorbers working conditions. $3^{\text {rd }}$ International Conference Trends in Agricultural Engineering, pp. 366-369. 